${ }^{1}$ King Abdullah University of Science and Technology,

${ }^{2}$ Faculty of Mathematics, Physics and Informatics, Comenius University, Bratislava, Slovakia,

${ }^{3}$ Earth Science Institute, Slovak Academy of Sciences, Bratislava, Slovakia

\title{
34 Abstract
}

We derive a theoretical parameter for three seismic scattering regimes where seismic

36 wavelengths are either much shorter, similar, or much longer than the correlation length of small-scale

37 Earth heterogeneities. We focus our analysis on the power spectral density of the von Karman

38 autocorrelation function, used to characterize the spatial heterogeneity of small-scale variations of elastic

39 rock parameters that cause elastic seismic wave scattering. Our theoretical findings are verified by

40 numerical simulations. We discover 1) that seismic scattering is proportional to the standard deviation of

41 velocity variations in all three regimes, 2) that scattering is inversely proportional to the correlation length

42 for the regime where seismic wavelengths are shorter than correlation length, but directly proportional

43 to the correlation length in the other two regimes, and 3) that scattering effects are weak due to

44 heterogeneities characterized by a gentle decay of the von Karman autocorrelation function for regimes

45 where seismic wavelengths are similar or much longer than the correlation length. 
Introduction

Heterogeneities in the Earth's crust and upper mantle cause seismic wave scattering, manifested

51 in so-called seismic coda waves that trail the main seismic phases. Often, coda waves are prominent

52 features of seismic recordings; they decay slowly with time, whereby the statistics of the temporal decay

53 provide information about the scattering process and the medium through which the waves travelled (e.g.

54 Aki 1969; Ritter et al., 1997; Sato and Fehler, 1998; Sato et al., 2012; Imperatori and Mai, 2013, 2015).

55 After Aki's (1969) interpretation that coda waves are back-scattered energy from uniformly distributed

56 heterogeneities in the Earth, several theoretical models were presented to explain seismic scattering, like

57 the single scattering model, the multiple scattering model, the diffusion model, or the energy-flux model

58 (Aki and Chouet, 1975; Sato, 1977; Gao et al., 1983; Frankel and Wennerberg, 1987). Additionally, the

59 coda envelope broadens with increasing travel distance due to wavefield scattering (Sato 2016), a process

60 that can be modelled employing a Markov approximation as stochastic treatment of the wave equation

61 in random media (Sato et al., 2012; Sato 2016). In contrast, S-wave coda excitation is mainly dominated

62 by scattering of direct S-waves from random heterogeneities in the Earth which can be modeled applying

63 the Born approximation (Sato et al., 2012; Sato and Emoto, 2017). In summary, coda waves are seismic-

64 wave energy trapped in the Earth due to the small-scale heterogeneities in the Earth.

Small-scale heterogeneities in the Earth can be described by a random spatial field superimposed onto a background homogeneous medium. For this purpose, several random-field models have been

67 proposed; these are conveniently characterized by an autocorrelation function (ACF). For example, von

68 Karman, Gaussian, exponential and Henyey-Greenstein ACF or a fractal distribution are used to describe

69 random fields of seismic wave velocity variations in the Earth (e.g. Frankel and Clayton, 1986; Holliger and

70 Levander, 1992; Sato and Fehler, 1998; Sato, 2019). Most commonly, the von Karman ACF is used (e.g. 
71 Hartzell et al. 2010; Imperatori and Mai, 2013; Bydlon and Dunham, 2015). The power spectral density

72 (PSD) of the von Karman AFC in three-dimension (3D) is given by

$$
p\left(k_{m}\right)=\frac{\sigma^{2}(2 \sqrt{\pi} a)^{3} \Gamma(H+1.5)}{\Gamma(H)\left(1+k_{m}^{2} a^{2}\right)^{(H+1.5)}}
$$

74 where $a, H, \sigma$ and $\Gamma$ are correlation length, Hurst exponent, standard deviation and the Gamma function,

75 respectively. We denote the wavenumber ( $2 \pi /$ wavelength) of medium heterogeneity by $k_{m}$, and of the

76 seismic wavefield by $k_{w}$, and write wavenumber $k$ in case $k_{m}$ and $k_{w}$ can be used interchangeably.

Several studies examined the range for correlation lengths, standard deviation, and Hurst exponent in the Earth, both in observational studies and numerical simulations. Frankel and Clayton

79 (1986) reported that velocity fluctuations with standard deviation of $5 \%$ and correlation lengths of $10 \mathrm{~km}$

80 (or greater) for 2D random media explain coda waves from micro earthquakes and travel time anomalies

81 across seismic arrays. Holliger (1996) obtained correlation lengths of 10 to 100 meters and Hurst exponent

82 in the range of $0.1-0.2$ by analyzing sonic logs. Ritter et al. (1998) estimated wave-velocity perturbations

83 of $3-7 \%$ and correlation length of $1-16 \mathrm{~km}$ for the lithosphere in central France. Recently, Sato (2019)

84 reported that velocity perturbations are $1-10 \%$ in the Earth's crust and upper mantle and that the Hurst

85 exponent typically falls in the range $0.0-0.5$, while correlation lengths vary widely depending on sample

86 size or dimension of the measurement system. Overall, standard deviation, Hurst exponent, and

87 correlation lengths are found to be in the range of $1-10 \%, 0.0-0.5$, and $1-15 \mathrm{~km}$, respectively.

Seismic wave scattering occurs as the elastic waves encounter spatial variations of elastic medium

89 properties. Whilst even the deterministic reflection of a seismic wave at an internal interface of a seismic

90 velocity contrast could be classified as "seismic scattering", the common nomenclature is that seismic

91 scattering is due to elastic-wave interactions with a spatially heterogeneous medium. In this context, the

92 (statistical) characteristics of the scattered wavefield depend on the stochastic properties of the medium. 
93 This concept is conveniently described considering the wavelengths $(\lambda)$ or wavenumbers $\left(k_{w}\right)$ of the elastic

94 wave, and characteristic scales (wavelengths) of the random media.

Based on wavelength $\lambda$ or wavenumber $k_{w}$ of the seismic wave, and the correlation length $a$ of

96 the random media, seismic wave scattering can be classified into three regimes: (i) $k_{w} \cdot a \gg 1(\lambda \ll a)$; (ii)

$97 k_{w} a \approx 1(\lambda \approx a)$; (iii) $k_{w} \cdot a \ll 1(\lambda \gg a)$ (Sato and Fehler, 1998; Sato et al., 2012;). The regime $k_{w} \cdot a \gg 1$

98 characterizes high-frequency scattering in which seismic wavelengths are much shorter than correlation

99 lengths. This regime is important for the earthquake engineering community in the context of high-

100 frequency $(10-20 \mathrm{~Hz})$ ground-shaking estimation, because seismic scattering redistributes seismic wave

101 energy (i.e. ground-motion amplitudes) in space and time. The regime $k_{w} \cdot a \approx 1$ represents the diffraction

102 condition, the most fundamental type of scattering. Finally, the regime $k_{w} \cdot a \ll 1$ denotes low-frequency

103 scattering for which seismic wavelengths are much longer than the correlation length of the random

104 medium. This regime is important for global seismology which uses primarily long wavelengths $(0.01-0.5$

$105 \mathrm{~Hz}$ ) to invert for the deterministic velocity structure of the Earth or earthquake source parameters (e.g.

106 centroid moment tensors).

Numerical and theoretical studies investigating the effects of seismic scattering on earthquake

108 ground-shaking suggest strong attenuation of ground-motion due to wavefield scattering (Shapiro and

109 Kneib, 1993; Mai, 2009; Hartzell et al., 2010; Imperatori and Mai, 2012, 2013; Yoshimoto et al., 2015; Vyas

110 et al., 2018). Bydlon and Dunham (2015) explained theoretically how the parameters describing the von

111 Karman ACF control wavefield scattering in 2D. Using numerical simulations, they verified that a

112 parameter $p_{0}=\sigma / a^{H}$ determines the nature of scattering in the $k_{w} \cdot a \gg 1$ limit, regardless of the specific

113 values of $\sigma$ and $a$. However, how the other parameters of the von Karman ACF $(a, \sigma$ and $H)$ affect 3D

114 seismic scattering has not been explored yet in detail. 
Here, we investigate seismic wave scattering in 3D and verify our theoretical results by numerical

116 simulations. First, we examine the mathematical expression for the power spectral density (PSD) of the

117 von Karman AFC (Eq. 1) to identify parameters that represent scattering behaviour in 3D for the three

118 different regimes, $k_{w} \cdot a \gg 1, k_{w} \cdot a \approx 1$ and $k_{w} \cdot a \ll 1$. Then we test our theoretical findings through numerical

119 simulations that cover the parameter space of these three regimes and allow us to examine how 120 scattering manifests itself in seismic waveforms and ground-motion amplitudes.

\section{Theory}

Bydlon and Dunham (2015) investigated high-frequency scattering $(f=1-30 \mathrm{~Hz})$ by considering

124 a 2D problem and the regime $k_{w} \cdot a \gg 1$. To analyze scattering under these assumptions, they simplified 125 the PSD of the von Karman ACF to obtain the root-mean-square (RMS) fluctuations of normalized seismic 126 wave velocity (wave speed), and then derived which parameters (i.e., $a, H$ and/or $\sigma$ ) control wavefield 127 scattering. Here, we extend their approach to 3D by considering three different $k_{w} \cdot a$ regimes.

Wavefield scattering is strongest if the wavenumber of the seismic wave is comparable to the wavenumber of heterogeneities in the medium. Hence, we simplify the PSD for the three regimes $\left(k_{w} \cdot a\right.$ $\gg 1, k_{w} \cdot a \approx 1$ and $\left.k_{w} \cdot a \ll 1\right)$ under the diffraction condition to obtain RMS of fluctuations of normalized

131 wave velocity (computed as the square root of the mean power, denoted as $P_{R M}$ ). By assuming the 132 diffraction condition, we derive theoretically the parameter $P_{R M}$, which in fact dictates the wavefield 133 scattering in 3D. Seismic scattering associated with a particular seismic wavelength will depend on the 134 amplitudes of velocity variations corresponding to that wavelength. However, we aim to understand the 135 overall wavefield scattering behaviour for a range of seismic wavelengths and heterogeneity scales in the 136 medium. Therefore, our $P_{R M}$ derivations are not only applicable for a monochromatic source or a single137 wavelength medium, but instead capture the broadband nature of scattering. Note that we only 
summarize the final equations for $P_{R M}$ for each regime in the main text; further details of the derivations

139 are provide in the Electronic supplement.

Regime I: $k_{w .} a \gg 1$

Our $P_{R M}$ derivation for this regime assumes that the source excites waves of equal amplitude (a

143 flat source spectrum) with wavenumbers from $k_{\min }$ to infinity, all of which interact with heterogeneities in

144 the medium with the same range of wavenumbers (albeit at different "intensity" or strength). Note that

145 this assumption is not completely satisfied in nature as earthquakes typically excite only a limited range

146 of frequencies, and not all of these frequencies will interact with the generally scale-limited medium

147 heterogeneities. However, the assumption allows us to calculate the overall wavefield scattering

148 behaviour for the regime $k_{w} \cdot a \gg 1$, for which seismic wavelengths are much shorter than the correlation

149 length of small-scale Earth heterogeneities. Then, the RMS fluctuations of normalized wave velocity $\left(P_{R M}\right)$

150 can be approximated by

$$
P_{R M}=\sqrt{\frac{1}{4 \pi} \int_{k_{\text {min }}}^{\infty} p(k) k^{2} d k} \approx \sqrt{\left(c_{0}+c_{1} H+c_{2} H^{2}\right)} \frac{\sigma}{a^{H}} \frac{\pi^{1 / 4}}{k_{\min }^{H}}
$$

152 Therefore, the $P_{R M}$ dependency is given by,

$$
P_{R M} \propto \sqrt{\left(c_{0}+c_{1} H+c_{2} H^{2}\right)} \frac{\sigma}{a^{H}}
$$

154 where we approximate the term depending on $H$ by a quadratic function (with coefficients $c_{0}=0.89, c_{1}=$

155 0.53, and $c_{2}=-0.08$; see Fig. S1a and derivation in electronic supplement for details). Note that we 156 characterize the scattering behavior for the entire regime $k_{w} \cdot a \gg 1$, rather than for a particular wavelength

157 in this regime by using integration limits in Eq. 2 from $k_{\min }$ to infinity, and not over any arbitrary

158 wavenumber range. Therefore, the parameter $P_{R M}$ (Eq. 3) becomes independent of wavenumber. 
159 Comparing Eq. 3 with parameter $p_{0}=\sigma / a^{H}$ (Bydlon and Dunham, 2015) reveals that even in the regime

$160 k_{w} \cdot a \gg 1$, scattering in 3D is more complex than in 2D. Eq. 3 illustrates that in the high-frequency scattering

161 regime, (a) scattering is proportional to the standard deviation of the velocity fluctuations, (b) scattering

162 is inversely proportional to the correlation length $a$, and c) the Hurst exponent has a strongly non-linear

163 effect on scattering. Interestingly, if the Hurst exponent approaches its theoretical lower limit of zero

$164(\mathrm{H} \rightarrow 0)$, Eq. 3 can be further simplified to

$$
P_{R M} \propto \sigma,
$$

indicating that scattering is controlled by the standard deviation of the velocity variations in this case.

Regime II: $k_{w} \cdot a \approx 1$

We assume that the source excites waves having a flat source spectrum with wavenumbers from

$170 k_{1}$ to $k_{2}$, all of which interact with medium heterogeneities of the same wavenumber range. If seismic

171 wavelengths are comparable to the correlation length of heterogeneities, the RMS fluctuations of

172 normalized wave velocity can be approximated by

$$
P_{R M}=\sqrt{\frac{1}{4 \pi} \int_{k_{1}}^{k_{2}} p(k) k^{2} d k} \approx\left(\frac{\pi}{18}\right)^{1 / 4} a^{3 / 2} \sigma \sqrt{\left(c_{1} H+c_{2} H^{2}\right)} \sqrt{\left(k_{2}^{3}-k_{1}^{3}\right)}
$$

174 Therefore, the $P_{R M}$ dependency is given by,

$$
P_{R M} \propto \sqrt{\left(c_{1} H+c_{2} H^{2}\right)} a^{3 / 2} \sigma,
$$

176 Where coefficients are given as $c_{1}=0.93$, and $c_{2}=-0.27$ (see Fig. S1b). Analyzing Eq. 6 for $P_{R M}$ reveals that

177 a) scattering is proportional to $\sigma$, similar to the regime $k_{w} \cdot a \gg 1$, b) scattering is proportional to correlation

178 length $a$, in contrast to regime $k_{w} . a \gg 1$ (compare Eq. 6 with Eq. 3), and c) scattering is correlated with the

179 Hurst exponent (as $H$ approaches zero, scattering effects weaken and become eventually negligible). 
Regime III: $k_{w} \cdot a \ll 1$

Here, we assume that the source excites waves of equal amplitude (a flat source spectrum) with

wavenumbers from zero to $k_{1}$, all of which interact with medium heterogeneities. If seismic wavelengths wave velocity can be approximated by

$$
P_{R M}=\sqrt{\frac{1}{4 \pi} \int_{0}^{k_{1}} p(k) k^{2} d k} \approx\left(\frac{4 \pi}{9}\right)^{1 / 4} a^{3 / 2} \sigma \sqrt{\left(c_{1} H+c_{2} H^{2}\right)} k_{1}^{3 / 2}
$$

$$
P_{R M} \propto \sqrt{\left(c_{1} H+c_{2} H^{2}\right)} a^{3 / 2} \sigma,
$$

where coefficients $c_{1}=0.93$, and $c_{2}=0.40$ (see Fig. S1c). Note that only constant $c_{2}$ is different between

Eq. 8 and Eq. 6 , therefore, $P_{R M}$ for the regime $k_{w} . a \ll 1$ is similar to that for $k_{w} \cdot a \approx 1$, except that the effect of $H$ on scattering is stronger for $k_{w} \cdot a \ll 1$ than for $k_{w} \cdot a \approx 1$ because $c_{2}>0$ (compare Eq. 6 and Eq. 8).

\section{Verification of Theory by Simulations}

In this section, we verify our findings (Eq. 3, 4, 6, 8) by conducting seismic wavefield simulations

in random media. Since our simulations do not strictly satisfy the assumptions used for the derivations of

$196 P_{R M}$, we validate only proportionality or inverse-proportionality of $P_{R M}$ with correlation length, standard

197 deviation, and Hurst exponent, rather than the complete expressions (Eq. 3, 4, 6, 8). To numerically test

198 our results for the three scattering regimes, we fix the correlation length $a$ and modify the source

199 frequency to radiate seismic waves with different frequencies (i.e., we are altering the wavenumber $k_{w}$ ).

200 For computing synthetic seismograms, we use a generalized 3D finite-difference method with second- 
order accuracy in space and time (SORD code by Ely et al., 2008). Our simulations consider several

202 discretized Earth models, a point-source earthquake model, and receiver locations at which ground203 motions are stored. We then analyze waveforms and peak ground acceleration (PGA), and confront the 204 numerical results with our theoretical analysis.

\section{Set up for Numerical Modeling}

We consider a point source (moment magnitude $\mathrm{M}_{\mathrm{w}} \sim 2.84$ ) at a depth of $7.5 \mathrm{~km}$, with strike, dip,

208 and rake of $22.5^{\circ}, 90^{\circ}$, and $0^{\circ}$, respectively. The source-time function (STF) is a Gaussian. We define STFs

209 to radiate frequencies required to properly sample the three regimes $\left(f_{\max }=5.0 \mathrm{~Hz}\right.$ for $k_{w} \cdot a \gg 1, f_{\max }=0.5$

$210 \mathrm{~Hz}$ for $k_{w} \cdot a \approx 1$ and $f_{\max }=0.03 \mathrm{~Hz}$ for $k_{w} \cdot a \ll 1$, see Fig S2; $f_{\max }$ is the high frequency limit of the flat portion

211 of the slip velocity spectrum). For example, a point source radiating frequencies of $5.0 \mathrm{~Hz}, 0.5 \mathrm{~Hz}$ and 0.03

$212 \mathrm{~Hz}$ in a heterogeneous medium with background shear-wave velocity $3.464 \mathrm{~km} / \mathrm{s}$ and stochastic

213 perturbations with correlation length of $1 \mathrm{~km}$ yields $k_{w} \cdot a \approx 9.0,0.9$ and 0.05 , respectively.

To create a velocity model with small-scale heterogeneities, we add random-field variations of

215 seismic wave velocities, characterized by an isotropic von Karman ACF, to the uniform background Earth 216 model (with S-wave velocity $3464 \mathrm{~m} / \mathrm{s}$, P-wave velocity, $6000 \mathrm{~m} / \mathrm{s}$, and density $2700 \mathrm{~kg} / \mathrm{m}^{3}$ ). In total, we

217 generate twelve 3D computational models (M1 to M12; Table 1), considering three correlation lengths $218(1.0 \mathrm{~km}, 5.0 \mathrm{~km}, 10.0 \mathrm{~km})$, two values of standard deviation $(5 \%, 10 \%)$, and two Hurst exponents $(0.1,0.5)$.

219 For each combination of medium parameters, we create one realization of random inhomogeneity in S-

220 wave speed, P-wave speed, and density. S-wave velocity distributions at the surface are shown for all

221 twelve computational models (Figs 1a, 1b). Theoretical 1D power spectra for seven selected models are 222 plotted to illustrate effects of correlation lengths, standard deviation, and Hurst exponent on the spectral 
shape (Fig 1c). Power spectra for two specific models, $M 2$ and $M 11$, are examined for the three scattering

224 regimes considering the three STFs used in this study (Fig. 1d).

The size of the computational domain must be chosen such that seismic waves propagate to largeenough distances that ensure sufficient wave interaction with medium heterogeneities to develop scattering. At the same time, the domain should be as small as possible to minimize computational cost. Given these constraints, we define different computational domain sizes and grid spacings, depending on scattering regime. For the regime $k_{w} \cdot a \gg 1$, we use grid spacing $\mathrm{h}=25 \mathrm{~m}(\mathrm{dt}=0.0015 \mathrm{~s}$ ) on a domain of $60 \times 60 \times 15 \mathrm{~km}$, allowing travel distance of $\sim 40$ wavelengths (at $f=5.0 \mathrm{~Hz}$ ). Combining these models with STF1 (Fig. S2a) yields $k_{w} \cdot a$ in the range of 9 to 90 . For $k_{w} \cdot a \approx 1$ we use $\mathrm{h}=75 \mathrm{~m}(\mathrm{dt}=0.0045 \mathrm{~s}$ ) and a larger domain, $355 \times 355 \times 30 \mathrm{~km}$, corresponding to travel distance of $\sim 50$ wavelengths (at $f=0.5 \mathrm{~Hz}$ ). The eight corresponding models are denoted by the suffix "-L" (see Tab 1 and Fig S3) and when combined with STF2

234 (Fig. S2b), they result in $k_{w} \cdot a$-values between 0.9 and 4.5 . For $k_{w} \cdot a \ll 1$, we use $\mathrm{h}=1000 \mathrm{~m}(\mathrm{dt}=0.055 \mathrm{~s}$ ) and an extra-large domain, $2000 \times 2000 \times 60 \mathrm{~km}$ (ignoring the spherical nature of Earth), denoted by the suffix "-EL" (see Tab 1 and Fig S4). When combined with STF3 (Fig. S2c), the corresponding $k_{w} \cdot a$ values fall in the range 0.27 to 0.5 . Owing to the very long wavelengths in this regime $(\sim 115 \mathrm{~km}$ at $f=0.03 \mathrm{~Hz})$, the domain

238 allows travel distances of only 15 wavelengths, significantly lower than those in the two previous 239 regimes. However, the cost for computational models allowing travel distances of $\sim 45-50$ wavelengths 240 would be exorbitant. In total, we use 28 computational models with random inhomogeneities, twelve of

241 which are for $k_{w} \cdot a \gg 1$, eight for $k_{w} \cdot a \approx 1$ and eight for $k_{w} \cdot a \ll 1$ regimes. Our simulations consumed nearly 242 four million core-hours of computational resources on a Cray XC40 supercomputer. To establish a base 243 case for comparison, we also conduct simulations in a homogeneous medium for each regime. 
247 it is in the lower left corner. Receiver geometry and epicenter location are designed to obtain the best

248 possible azimuthal coverage of stations and to allow for sufficiently large travel distances for seismic 249 waves to develop scattering, at the same time also minimizing computational costs. Virtual stations are 250 distributed along rings with radial spacing of $0.1,0.2$ and $3.5 \mathrm{~km}$, for $k_{w} \cdot a \gg 1, k_{w} \cdot a \approx 1$ and $k_{w} \cdot a \ll 1$ 251 regimes, respectively. Therefore, each ring (arc) of stations contains a different number of stations at 252 different azimuths. The smallest ring (arc) used for PGA statistics has 314 (radius 5km), 196 (radius $25 \mathrm{~km}$ )

253 and 134 (radius $300 \mathrm{~km})$ stations for the three regimes $\left(k_{w} \cdot a \gg 1 ; k_{w} \cdot a \approx 1 ; k_{w} \cdot a \ll 1\right)$. Therefore, our

254 receiver geometry is statistically independent and PGA statistics are robust. All waveforms are low-pass 255 filtered using a fourth-order Butterworth filter with cutoff frequencies of $5 \mathrm{~Hz}, 0.5 \mathrm{~Hz}$ and $0.03 \mathrm{~Hz}$ for the 256 three scattering regimes, respectively.

\section{Quantifying Seismic Scattering in Numerical Results}

Seismic scattering redistributes energy in space and time from direct P- and S-waves into the latearriving coda waves. Consequently, peak ground acceleration (PGA) in a homogeneous medium will be, 261 on average, higher than in a scattering medium. Therefore, we examine ratios of PGA-values to quantify 262 scattering "strength" in numerical simulations. Horizontal components of acceleration are mostly used in 263 earthquake engineering applications (e.g., Boore and Atkinson, 2008; Chiou and Youngs, 2008), because 264 wave amplitudes on the vertical component are usually smaller than on the horizontal components. 265 Therefore, we analyze horizontal PGA (computed as maximum magnitude of acceleration from the two 266 horizontal components). We illustrate scattering effects and resulting PGA values by comparing 267 waveforms for selected receivers s1, s2 and s3 (see Fig 1a for their locations).

In Fig 2 we compare horizontal-component ground-acceleration waveforms at selected stations 
270 (models M3 and M6) with those for the homogeneous medium PGA values are consistent with our

271 expectation that stronger scattering leads to lower PGA. In this particular case, the scattering for model

272 M3 is weaker than for model M6 (see also acceleration snapshots in Fig S5). Additionally, ground

273 acceleration comparison for M6 at three stations (Fig S6) shows prominent coda evolution and reduced

274 maximum acceleration values as epicentral distance increases (from $s 4$ to $s 6$ ). Fig $2 b$ reveals that

275 waveforms for two models with different correlation lengths ( $M 1$ and M3) are almost identical, with only

276 small time shifts. This indicates that the two models yield almost identical levels of scattering (confirmed

277 also by comparing acceleration snapshots for M1 and M3 in Fig S5). Correspondingly, PGA values are

278 comparable. In addition, these comparisons (Figs $2 \mathrm{a}$ and $2 \mathrm{~b}$ ) suggest that scattering is primarily controlled

279 by the standard deviation of the medium heterogeneities, whereas the correlation length has a negligible

280 effect for a small $H$ value $(H=0.1)$, consistent with our theoretical analysis in Eq. 4. However, we note that

281 PGA only works well in such comparisons because we computed a reference solution for the

282 homogeneous medium. Without such a reference case, interpreting PGA values directly as indicator for

283 "scattering strength" would be misleading.

\section{Statistical Analysis of Scattering}

Next, we calculate the mean and standard deviation of PGA values for all stations at a given

287 epicentral distance and for a given computational model (see Fig. S7 for a comparative summary of all 288 computational models). To estimate the average scattering-related PGA reduction at a given epicentral 289 distance, we define the "mean PGA ratio" (MPR), at a particular epicentral distance, as the ratio between 290 the mean PGA values from any heterogeneous Earth model to the mean PGA-values from the reference 291 homogeneous Earth model. As epicentral distance increases, the MPR is expected to decrease because 292 the redistribution of seismic energy due to scattering is cumulative with propagation distance. 
Figure 3 summarizes our results for $k_{w} \cdot a \gg 1$. For $H=0.1$ we find the MPRs for models with $\sigma=$ $29410 \%(M 4, M 5, M 6)$ are lower than for models with $\sigma 5 \%$ (M1, M2, M3) (Fig. 3a). At the same time, MPRs 295 of both groups are very similar, supporting our theoretical conclusion that for small $\mathrm{H}$ the correlation 296 length has insignificant effects on scattering, which in this regime is controlled by standard deviation (Eq.

297 4). The apparent plateau in MPRs for distances 10 to $20 \mathrm{~km}$ is a consequence of source effects being 298 masked by wavefield scattering effects due to the hypocenter location (see Fig. S8 for more details on the 299 effects of hypocentral depths on MPRs). Fig $3 b$ compares solutions for $H=0.5$, for which we expect a 300 significant effect of both correlation length and standard deviation. For fixed $\sigma$, we observe that the MPR's 301 for models with shorter correlation length are lower than those with longer correlation length $\left(M P R_{M 7}<\right.$ $302 M P R_{M 8}<M P R_{M 9}$; similarly $\left.M P R_{M 10}<M P R_{M 11}<M P R_{M 12}\right)$. This finding is consistent with our conclusion 303 that scattering is inversely proportional to correlation length for large H (Eq. 3). Also, MPR's for models 304 with $\sigma=10 \%$ are lower than those for corresponding models with $\sigma=5 \%\left(M P R_{M 10}<M P R_{M 7}, M P R_{M 11}<\right.$ $\left.305 M P R_{M 8}, M P R_{M 12}<M P R_{M 9}\right)$, demonstrating that scattering is proportional to the standard deviation of 306 velocity variations for large $H$. Thus, these observations validate our theoretical conclusions for the regime $307 \quad k_{w} \cdot a \gg 1$. for models with shorter correlation length are higher than MPR's for models with longer correlation length $\left(M P R_{M 1-L}>M P R_{M 2-L}, M P R_{M 4-L}>M P R_{M 5-L}, M P R_{M 7-L}>M P R_{M 8-L}, M P R_{M 10-L}>M P R_{M 11-L}\right)$

311 revealing that scattering is proportional to correlation length (see Fig. 4a and Fig. 4b). The MPR's for 312 models with $\sigma=5 \%$ are higher than those for model with $\sigma=10 \%\left(M P R_{M 1-L}>M P R_{M 4-L}, M P R_{M 2-L}>\right.$ $\left.313 M P R_{M 5-L}, M P R_{M 7-L}>M P R_{M 10-L}, M P R_{M 8-L}>M P R_{M 11-L}\right)$, indicating that scattering is proportional to 314 the standard deviation of velocity fluctuations. The MPR's for models with $H=0.1$ are larger than those 315 for models with $H=0.5\left(M P R_{M 1-L}>M P R_{M 7-L}, M P R_{M 2-L}>M P R_{M 8-L}, M P R_{M 4-L}>M P R_{M 10-L}\right.$, 
$\left.316 M P R_{M 5-L}>M P R_{M 11-L}\right)$, therefore, scattering is proportional to the Hurst exponent $H$. These 317 observations are also consistent with our theoretical findings for $k_{w} \cdot a \approx 1$ (see Eq. 6).

$318 \quad$ Finally, we show MPR statistics for the regime $k_{w} \cdot a \ll 1$ (Figure 5). First, recall that due to 319 prohibitively large computational costs we used a smaller computational domain (see Section Set up for

320 Numerical Modeling). Consequently, scattering is less well developed for $k_{w} \cdot a \ll 1$, and hence effects on 321 MPR's are not as pronounced as in the other two regimes. Still, the effects are strong enough to support 322 our theoretical derivation (see waveform comparison in Fig. S9 and station locations in Fig. S4). The MPR's 323 for models with $10 \mathrm{~km}$ correlation length are lower than those for $5 \mathrm{~km}$ correlation length $\left(M P R_{M 3-E L}<\right.$ $324 M P R_{M 2-E L}, M P R_{M 6-E L}<M P R_{M 5-E L}, M P R_{M 9-E L}<M P R_{M 8-E L}, M P R_{M 12-E L}<M P R_{M 11-E L}$ ), showing 325 that scattering is proportional to correlation length. The MPR's for models with $\sigma=10 \%$ are lower than 326 those for $\sigma=5 \%\left(M P R_{M 12-E L}<M P R_{M 9-E L}, M P R_{M 11-E L}<M P R_{M 8-E L}\right)$, suggesting that scattering is also 327 proportional to the standard deviation of velocity variations. These observations agree well with our 328 theoretical considerations for $k_{w} a \ll 1$ (see Eq. 8).

In summary, our results from numerical simulations are consistent with our conclusions based on theoretical derivation for all three considered scattering regimes.

\section{Discussion and Conclusions}

We derive a new parameter $P_{R M}$ to quantify 3D seismic wavefield scattering. $P_{R M}$ is based on the 334 assumption that small-scale heterogeneities in seismic velocity are characterized by the von Karman ACF. $335 P_{R M}$ helps to understand the influence of the parameters of the von Karman ACF on seismic scattering for 336 three considered regimes $\left(k_{w} \cdot a \gg 1, k_{w} \cdot a \approx 1\right.$ and $\left.k_{w} \cdot a \ll 1\right)$. We test our theoretical consideration through 
statistical analysis of a suite of numerical simulations that capture seismic scattering in different scattering

338 regimes.

We find that the strength of wavefield scattering in all three regimes is proportional to the standard deviation of heterogeneities. Seismic scattering is also proportional to the correlation length in

341 the regimes $k_{w} \cdot a \approx 1$ and $k_{w} \cdot a \ll 1$, but for the regime $k_{w} \cdot a \gg 1$ the scattering is inversely proportional to

342 correlation length. For regime $k_{w} \cdot a \gg 1$, we also find that if the Hurst exponent $\mathrm{H}$ approaches zero,

343 scattering will be controlled solely by standard deviation. However, for $k_{w} \cdot a \approx 1$ and $k_{w} \cdot a \ll 1$, scattering

344 is weakly impacted for small values of $H$, with scattering vanishing in the limit of $H \rightarrow 0$. wavenumber $k_{m}$,

$$
\int_{0}^{\infty} p\left(k_{m}\right) d k_{m}=4 \pi^{2} a^{2} \sigma^{2} H
$$

348 Eq. 9 represents the area under the power spectrum for a three dimensional isotropic PSD along one

349 wavenumber axis; it reveals that the area under the power spectrum depends on $a, H$ and $\sigma$, implying also

350 that the area under the power spectrum will be zero if any of $a$ or $H$ or $\sigma$ is zero. For example, M2 has

351 larger area under the power spectrum than M1 due to larger correlation lengths of $\mathrm{M} 2$, although standard

352 deviation and Hurst exponent are identical for M1 and M2 (see Fig. 1c). The area under the power

353 spectrum can be linked to wavefield scattering as it represents the total scattering power of the

354 heterogeneous medium in terms of the sum of amplitude squares of seismic-velocities. Correspondingly,

355 in the limit of any of the von Karman parameters approaching zero, wavefield scattering will become 356 negligible. 
explained by the amplitude and shape of the PSD. The standard deviation scales the power spectra

360 without changing the shape of the power spectra (hence, area under the power spectra), resulting in 361 scattering proportional to $\sigma$ for all three regimes $\left(k_{w} \cdot a \gg 1, k_{w} \cdot a \approx 1\right.$ and $\left.k_{w} \cdot a \ll 1\right)$. The tails of the power 362 spectra (decaying part) show inverse proportionality with correlation length a (e.g. compare tails of M7, $363 \mathrm{M} 8$ and M9 in Fig 1c), thus resulting in scattering being inversely proportional to $a$ for the regime $k_{w} \cdot a \gg$ 364 1. However, the plateau and corners (corner wavenumber $=2 \pi / a$ ) of the power spectra scale with 365 correlation length, leading to scattering being proportional to correlation length for $k_{w} \cdot a \ll 1$ and $k_{w} \cdot a \approx$ 366 1, respectively (e.g. compare plateau and corners of M7, M8 and M9 in Fig 1c). Furthermore, the plateau 367 and corner of power spectra grow as $H$ increases, therefore, scattering is proportional to $H$ for $k_{w} \cdot a \ll 1$ 368 and $k_{w} \cdot a \approx 1$. Fig $1 \mathrm{c}$ also shows that the tails of the power spectra tend to merge for small $H$ (see M1, M2 369 and $\mathrm{M} 3$ ) and diverge as $H$ increases (compare $M 7, M 8$ and $M 9$ ), implying a more complex dependency on $370 \mathrm{H}$ for scattering in the regime $k_{w} \cdot a \gg 1$. Hence, our findings can be explained by the shape and amplitude 371 of the PSD function of the von Karman ACF. Comparing our results for $k_{w} \cdot a \gg 1$ for the $3 \mathrm{D}$ problem (Eq. 3) with the $2 \mathrm{D}$ results by Bydlon and 373 Dunham (2015) $\left(p_{0}=\sigma / a^{H}\right)$ reveals that the effect of standard deviation and correlation length remains 374 the same, but the effect of the Hurst exponent $H$ is stronger in 3D. However, if the Hurst exponent 375 approaches zero, scattering effects are dominated by standard deviation, both in 2D and 3D. This is an 376 important finding, since values of $H$ smaller than 0.5 have been reported by Sato (2019) for the Earth's 377 crust and mantle. Here we propose to quantify the overall wavefield scattering directly via an integral of the PSD 379 function of the random media. We note that Sato et al. (2012) analyzed a plane wave scattered by a 380 localized inhomogeneity using the wave equation. They solved the wave equation utilizing Born 381 approximation, i.e., they assumed that the amplitude of velocity variations is negligibly small compared 
382 to background velocity, that the amplitude of the scattered wavefield is negligibly small compared to the 383 amplitude of incident wavefield, and that the scattered wavefield has only a small phase change after 384 passing through the heterogeneity. Therefore, derivations by Sato et al., (2012) are valid for high 385 frequency scattering, when seismic wavelengths are very short compared to the length scales of medium 386 heterogeneity. They found that the scattering coefficient depends on the PSD function of the random 387 media as follows (Eq. 4.25 from Sato et al., 2012),

$$
g(\theta, \omega)=\frac{k_{w}^{4}}{\pi} P\left(2 k_{w} \sin \frac{\theta}{2}\right)
$$

In Eq. 10, $\theta$ is the angle between incident and scattered waves; $\omega$ and $k_{w}$ are angular frequency and wavenumber of the incident wavefield, respectively. The scattering coefficient reveals that a wave with wavenumber $k_{w}$ interacts with medium heterogeneities with wavenumber $k_{m}$, leading to

$$
k_{m}=2 k_{w} \sin \frac{\theta}{2}=2 \sin \frac{\theta}{2} k_{w}=C k_{w}
$$

$394=0$ ) and backward $(\theta=\pi)$ scattering, respectively. The average value of $C$ (over $\theta$ ) indicates the overall 395 interaction between $k_{m}$ and $k_{w}$, averaged over all directions. The average value of $C$ is 1.27 , therefore $k_{m}$ $396 \sim k_{w}$. This is consistent with our assumption for the derivation of $P_{R M}$, although we apply an ideal 397 diffraction condition $\left(k_{m}=k_{w}\right)$. Note that our $P_{R M}$ results will not change even if we use a more relaxed 398 diffraction condition (i.e. $k_{m} \sim k_{w}$ ). Hence, our theory complies with Sato et al. (2012), but taking a different 399 perspective on evaluating the wavefield scattering. Note that the detailed theoretical analysis to fully 400 describe the wavefield scattering in 3D requires considering the $3 \mathrm{D}$ elastic wave equation with complex 401 earthquake source characteristics (radiated wavefield) in 3D random media with anisotropic wave 402 propagation. This derivation is beyond the scope of the present study. 
In summary, our theoretical analysis of the von Karman PSD, used to represent random spatial

404 variation in seismic wave velocities and rock density, helps to develop a physics-based understanding of

405 how standard deviation, correlation length, and Hurst exponent govern three-dimensional seismic

406 wavefield scattering for three scattering regimes $\left(k_{w} \cdot a \gg 1, k_{w} \cdot a \approx 1\right.$ and $\left.k_{w} \cdot a \ll 1\right)$. This will help studies

407 on ground-motion simulations for earthquake shaking as well as research on global seismic wave

408 propagation in 3D Earth models to properly simulate elastic wavefield scattering.

410 Data and Resources

Ground-motions simulations carried out to verify the outcomes of theoretical derivation

412 generated nearly 2.5 TB of data which can be provided via personal communication. This manuscript has

413 an electronic supplement which comprises the complete derivation of the root-mean-square fluctuations

414 of normalized wave velocity using power spectral density of the von Karman autocorrelation function for

415 three scattering regimes $\left(k_{w} \cdot a \gg 1, k_{w} \cdot a \approx 1\right.$ and $\left.k_{w} \cdot a \ll 1\right)$. The electronic supplement also contains figures

416 of the quadratic fit to ratios of gamma functions, three Gaussian source time functions, simulations setup

417 depicting receiver geometry and S-wave speed variations, acceleration waveforms comparison from few

418 receivers, snapshots of ground-acceleration wavefield at Earth surface and peak ground acceleration 419 statistics.

\section{Acknowledgements}

422 The research presented in this article is supported by King Abdullah University of Science and Technology

423 (KAUST) in Thuwal, Saudi Arabia, grants BAS/1/1339-01-01 and URF/1/3389-01-01. MG was partially

424 supported by Scientific Grant Agency VEGA, grant 2/0046/20. Earthquake ground-motion simulations 
425 have been carried out using the KAUST Supercomputing Laboratory (KSL), and we acknowledge the

426 support of the KSL staff. We thank Art Frankel and an anonymous reviewer, as well as the editor Adrien

427 Oth, for their constructive critical review that helped us to improve the manuscript.

428

429 References

430 Aki, K. (1969). Analysis of the seismic coda of local earthquakes as scattered waves. Journal of 431 geophysical research, 74(2), 615-631.

Aki, K., \& Chouet, B. (1975). Origin of coda waves: source, attenuation, and scattering effects.

433 Journal of geophysical research, 80(23), 3322-3342.

Boore, D. M., \& Atkinson, G. M. (2008). Ground-motion prediction equations for the average

435 horizontal component of PGA, PGV, and 5\%-damped PSA at spectral periods between $0.01 \mathrm{~s}$ and $10.0 \mathrm{~s}$.

436 Earthquake Spectra, 24(1), 99-138.

Bydlon, S. A., \& Dunham, E. M. (2015). Rupture dynamics and ground motions from earthquakes

438 in 2-D heterogeneous media. Geophysical Research Letters, 42(6), 1701-1709.

440 ground motion and response spectra. Earthquake Spectra, 24(1), 173-215.

441 Ely, G. P., Day, S. M., \& Minster, J. B. (2008). A support-operator method for viscoelastic wave 442 modelling in 3-D heterogeneous media. Geophysical Journal International, 172(1), 331-344.

443 Frankel, A., \& Clayton, R. W. (1986). Finite difference simulations of seismic scattering:

444 Implications for the propagation of short-period seismic waves in the crust and models of crustal 445 heterogeneity. Journal of Geophysical Research: Solid Earth, 91(B6), 6465-6489. 
447 and intrinsic attenuation. Bulletin of the Seismological Society of America, 77(4), 1223-1251.

449 three-dimensional medium. pure and applied geophysics, 121(1), 3-15.

451 perturbations on predicted ground motions. Bulletin of the Seismological Society of America, 100(4), $452 \quad 1415-1426$.

Holliger, K. (1996). Upper-crustal seismic velocity heterogeneity as derived from a variety of P-

454 wave sonic logs. Geophysical Journal International, 125(3), 813-829.

456 from the Ivrea zone. Geophysical Research Letters, 19(11), 1153-1156.

Imperatori, W., \& Mai, P. M. (2012). Sensitivity of broad-band ground-motion simulations to earthquake source and Earth structure variations: an application to the Messina Straits (Italy). Geophysical 459 Journal International, 188(3), 1103-1116.

461 dimensional scattering media. Geophysical journal international, 192(2), 725-744.

463 on near-source scattering and ground-motion variability. Geophysical Journal International, 202(3), 2163-

4642181. 
469 crust observations in the Massif Central, France. Physics of the earth and planetary interiors, 104(1-3), $470 \quad 127-146$.

Ritter, J. R., Shapiro, S. A., \& Schechinger, B. (1998). Scattering parameters of the lithosphere 472 below the Massif Central, France, from teleseismic wavefield records. Geophysical Journal International, 473 134(1), 187-198.

Sato H., \& Fehler, M. C. (1998). Seismic Wave Propagation and Scattering in the Heterogenous 475 Earth, , Springer-Verlag, New York, ISBN \#0-387-98329-5.

476 Sato, H. (1977). Single isotropic scattering model including wave conversions simple theoretical 477 model of the short period body wave propagation. Journal of Physics of the Earth, 25(2), 163-176.

478 Sato, H. (2016). Envelope broadening and scattering attenuation of a scalar wavelet in random 479 media having power-law spectra. Geophysical Journal International, 204(1), 386-398.

Sato, H. (2019). Power spectra of random heterogeneities in the solid earth. Solid Earth, 10(1), $481 \quad 275-292$.

Sato, H., \& Emoto, K. (2017). Synthesis of a scalar wavelet intensity propagating through von 483 Kármán-type random media: joint use of the radiative transfer equation with the Born approximation and 484 the Markov approximation. Geophysical Journal International, 211(1), 512-527.

485 Sato, H., Fehler, M. C., \& Maeda, T. (2012). Seismic wave propagation and scattering in the 486 heterogeneous earth. Springer Science \& Business Media. ISBN 978-3-642-23028-8. 
490 in the presence of source and medium heterogeneity. Geophysical Journal International, 214(3), 2035-

491 2052.Yoshimoto, K., Takemura, S., \& Kobayashi, M. (2015). Application of scattering theory to P-wave 492 amplitude fluctuations in the crust. Earth, Planets and Space, 67(1), 199.

493

494

495

496

497

498

499

500

501

502

503

504

505

506

507 
509 Author's full physical mailing addresses:

510 Dr. Jagdish Chandra Vyas,

511 Building 1, Level 3, office \#3110,

512 King Abdullah University of Science and Technology (KAUST),

513 Jeddah, Thuwal, 23955, Saudi Arabia.

514

515

516

517

Dr. Martin Galis,

518 Faculty of Mathematics, Physics and Informatics,

519 Comenius University,

520 Mlynska dolina F1,

52184248 Bratislava,

522 Slovakia.

523

524

525 Prof. Paul Martin Mai,

526 Building 1, Level 3, office \#3114,

527 King Abdullah University of Science and Technology (KAUST),

528 Jeddah, Thuwal, 23955, Saudi Arabia.

529

530

531

532

533

534

535

536

537 
539 Table 1: Parameters for the 28 computational 3D Earth models generated for this study.

\section{$541 \quad$ List of Figure Captions}

542 Figure 1: (a,b): S-wave speed distribution at the free surface for twelve 3D computational models for the

543 regime $k_{w} . a \gg 1$, generated using three correlation lengths $(1.0 \mathrm{~km}, 5.0 \mathrm{~km}, 10.0 \mathrm{~km})$, two standard

544 deviations $(5 \%, 10 \%)$ and two Hurst exponents $(0.1,0.5)$. The black star marks the epicenter. The sites

545 used for waveform comparison (black triangles, s1, s2, s3, s4, s5 and s6) and ground-motion analysis (black

546 dots in circular rings) are also shown. The beach ball shows the focal mechanism of the earthquake source.

547 Panels (a) and (b) depict random media with Hurst exponent 0.1 and 0.5 , respectively. (c): Theoretical 1-

548 D power spectra (PSD) for 3D Earth structure for seven selected models. Correlation length and Hurst

549 exponent alter the shape of the power spectra (solid lines), whereas standard deviation only scales the

550 PSD (mark dashed line; notice the scaling of M4 compared to M1, but their identical shape). (d): The

551 theoretical power spectra of the random media are constrained by the dimensions of the computational

552 model and the spatial grid size. The dashed and solid lines are spectra related to models M2 and M11,

553 whereas three different colors depict power spectra sampled according to the three scattering regimes.

555 Figure 2: Horizontal components (East-West, EW, and North-South, NS) of ground acceleration $\left(\mathrm{m} / \mathrm{s}^{2}\right)$ at 556 sites s1, s2, s3 (Fig 1a). Black dotted lines indicate theoretical P-and S-wave arrival times in the considered 557 homogeneous medium. Color-coded numbers indicate PGA values at individual sites. Waveforms are 558 normalized by their PGA-value in the homogeneous-medium simulations for a given site. (a) Illustration 
559 of scattering controlled by $\sigma$ for $k_{w} \cdot a \gg 1$ and small $H$; (b) Illustration of negligible effects of correlation

560 length on scattering for $k_{w} \cdot a \gg 1$ and small $H$.

562 Figure 3: Mean PGA ratios (MPR) for all twelve numerical simulations as a function of distance, depicting

563 the effects of wavefield scattering on ground-motions in the regime $k_{w} \cdot a \gg 1$. Panels (a) and (b) depict

564 MPR for media with $\mathrm{H}=0.1$ and $\mathrm{H}=0.5$, respectively. Grey dashed lines are plotted to facilitate the MPR

565 comparison in two nearby panels. Wavefield scattering is proportional to the standard deviation of

566 medium heterogeneities, and inversely proportional to correlation length for large Hurst exponent $(\mathrm{H}=$

$5670.5)$, but remains nearly unaffected by variations in correlation length for small Hurst exponent $(H=0.1)$.

568 The $k_{w} \cdot a$ maxima for correlation lengths of 1,5 and $10 \mathrm{~km}$ are 9.07, 45.36 and 90.72, respectively.

570 Figure 4: Mean PGA ratios (MPR) for eight numerical simulations as a function of distance, depicting the

571 effects of wavefield scattering on ground-motions in the regime $k_{w} \cdot a \approx 1$. Panels (a) and (b) depict MPR

572 for media with $\mathrm{H}=0.1$ and $\mathrm{H}=0.5$, respectively. Grey dashed lines are plotted to facilitate the MPR

573 comparison in two nearby panels. Wavefield scattering is proportional to correlation length, Hurst

574 exponent, and standard deviation of medium heterogeneities. The highest values of $k_{w} \cdot a$ for correlation

575 lengths of 1 and $5 \mathrm{~km}$ are 0.90 and 4.53, respectively.

577 Figure 5: Mean PGA ratios (MPR) for all eight numerical simulations as a function of distance, depicting

578 the effects of wavefield scattering on ground-motions in the regime $k_{w} \cdot a<1$. Panels (a) and (b) depict

579 MPR for media with $\mathrm{H}=0.1$ and $\mathrm{H}=0.5$, respectively. Grey dashed lines are plotted to facilitate the MPR

580 comparison in two nearby panels. Wavefield scattering is proportional to correlation length, Hurst 
581 exponent, and the standard deviation of medium heterogeneities. The highest values of $k_{w} \cdot a$ for 582 correlation lengths of 5 and $10 \mathrm{~km}$ are 0.27 and 0.54 , respectively. 


\section{List of Tables}

Table 1: Parameters for the 28 computational 3D Earth models generated for this study.

\begin{tabular}{|c|c|c|c|}
\hline Model Reference & $\begin{array}{l}\text { Correlation length } \\
\qquad a(\mathrm{~km})\end{array}$ & $\begin{array}{l}\text { Standard deviation } \\
\qquad \sigma(\%)\end{array}$ & $\begin{array}{c}\text { Hurst exponent } \\
\qquad\end{array}$ \\
\hline M1, M1-L & 1.0 & 5 & 0.1 \\
\hline M2, M2-L, M2-EL & 5.0 & 5 & 0.1 \\
\hline M3, M3-EL & 10.0 & 5 & 0.1 \\
\hline M4, M4-L & 1.0 & 10 & 0.1 \\
\hline M5, M5-L, M5-EL & 5.0 & 10 & 0.1 \\
\hline M6, M6-EL & 10.0 & 10 & 0.1 \\
\hline M7, M7-L & 1.0 & 5 & 0.5 \\
\hline M8, M8-L, M8-EL & 5.0 & 5 & 0.5 \\
\hline M9, M9-EL & 10.0 & 5 & 0.5 \\
\hline M10, M10-L & 1.0 & 10 & 0.5 \\
\hline M11, M11-L, M11-EL & 5.0 & 10 & 0.5 \\
\hline M12, M12-EL & 10.0 & 10 & 0.5 \\
\hline
\end{tabular}

Parameters of 28 computational 3D models generated using random fields characterized by von Karman autocorrelation functions (parametrized by correlation length, standard deviation and Hurst exponent). The suffixes "-L" and "-EL" indicate large and extra-large models, respectively. 


\section{List of Figures}

(a)
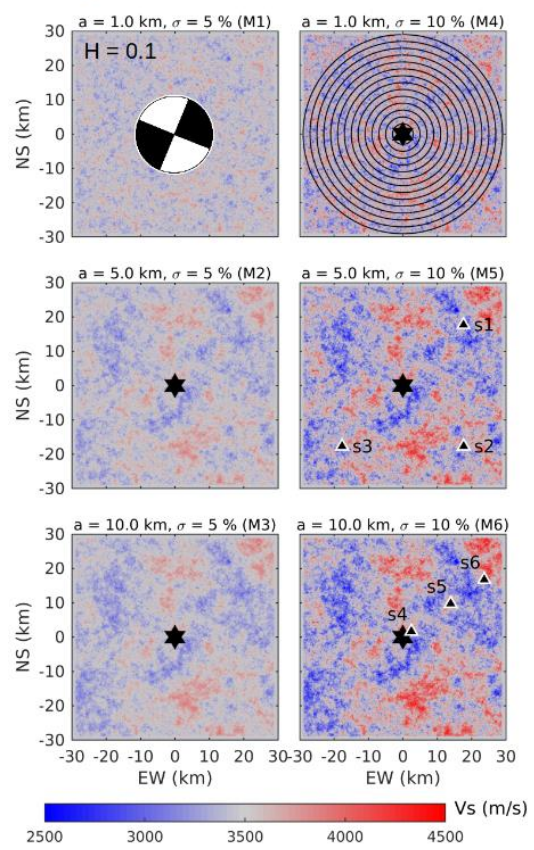

(c)

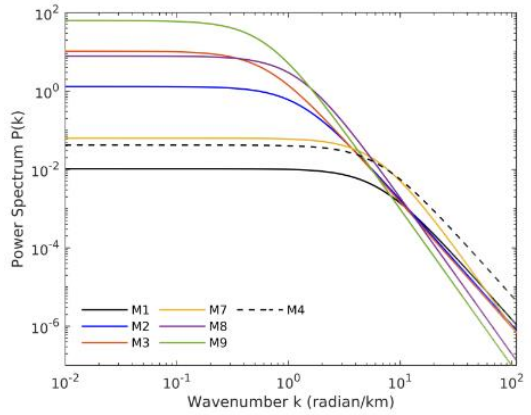

(b)

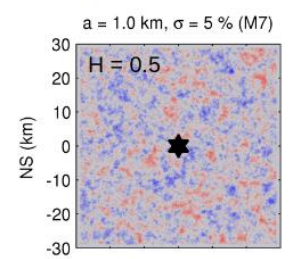

$=1.0 \mathrm{~km}, \sigma=10 \%(\mathrm{M} 10)$

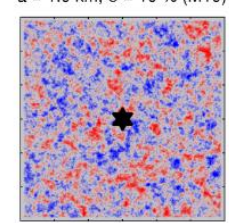

$=5.0 \mathrm{~km}, \sigma=5 \%(\mathrm{M} 8)$

$\mathrm{a}=5.0 \mathrm{~km}, \sigma=10 \%(\mathrm{M} 11)$

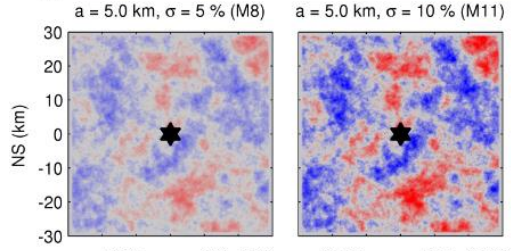

$\alpha=10.0 \mathrm{~km}, \sigma=5 \%(\mathrm{Mg}) \quad a=10.0 \mathrm{~km}, \sigma=10 \%(\mathrm{M} 12)$

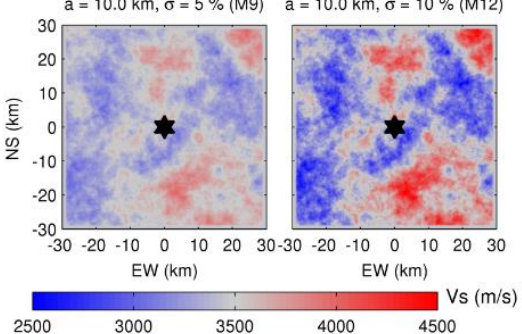

(d)

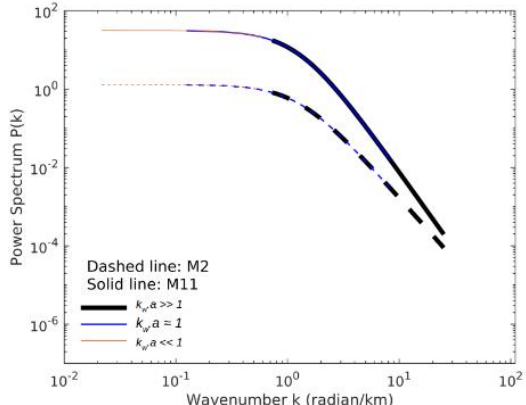

Figure 1: $(a, b)$ : $S$-wave speed distribution at the free surface for twelve 3D computational models for the regime $k_{w} \cdot a \gg 1$, generated using three correlation lengths $(1.0 \mathrm{~km}, 5.0 \mathrm{~km}, 10.0 \mathrm{~km})$, two standard deviations $(5 \%, 10 \%)$ and two Hurst exponents $(0.1,0.5)$. The black star marks the epicenter. The sites used for waveform comparison (black triangles, s1, s2, s3, s4, s5 and s6) and ground-motion analysis (black dots in circular rings) are also shown. The beach ball shows the focal mechanism of the earthquake source. Panels (a) and (b) depict random media with Hurst exponent 0.1 and 0.5 , respectively. (c): Theoretical 1-D power spectra (PSD) for 3D Earth structure for seven selected models. Correlation length and Hurst exponent alter the shape of the power spectra (solid lines), whereas standard deviation only scales the PSD (mark dashed line; notice the scaling of M4 compared to M1, but their identical shape). (d): The theoretical power spectra of the random media are constrained by the dimensions of the computational model and the spatial grid size. The dashed and solid lines are spectra related to models M2 and M11, whereas three different colors depict power spectra sampled according to the three scattering regimes. 

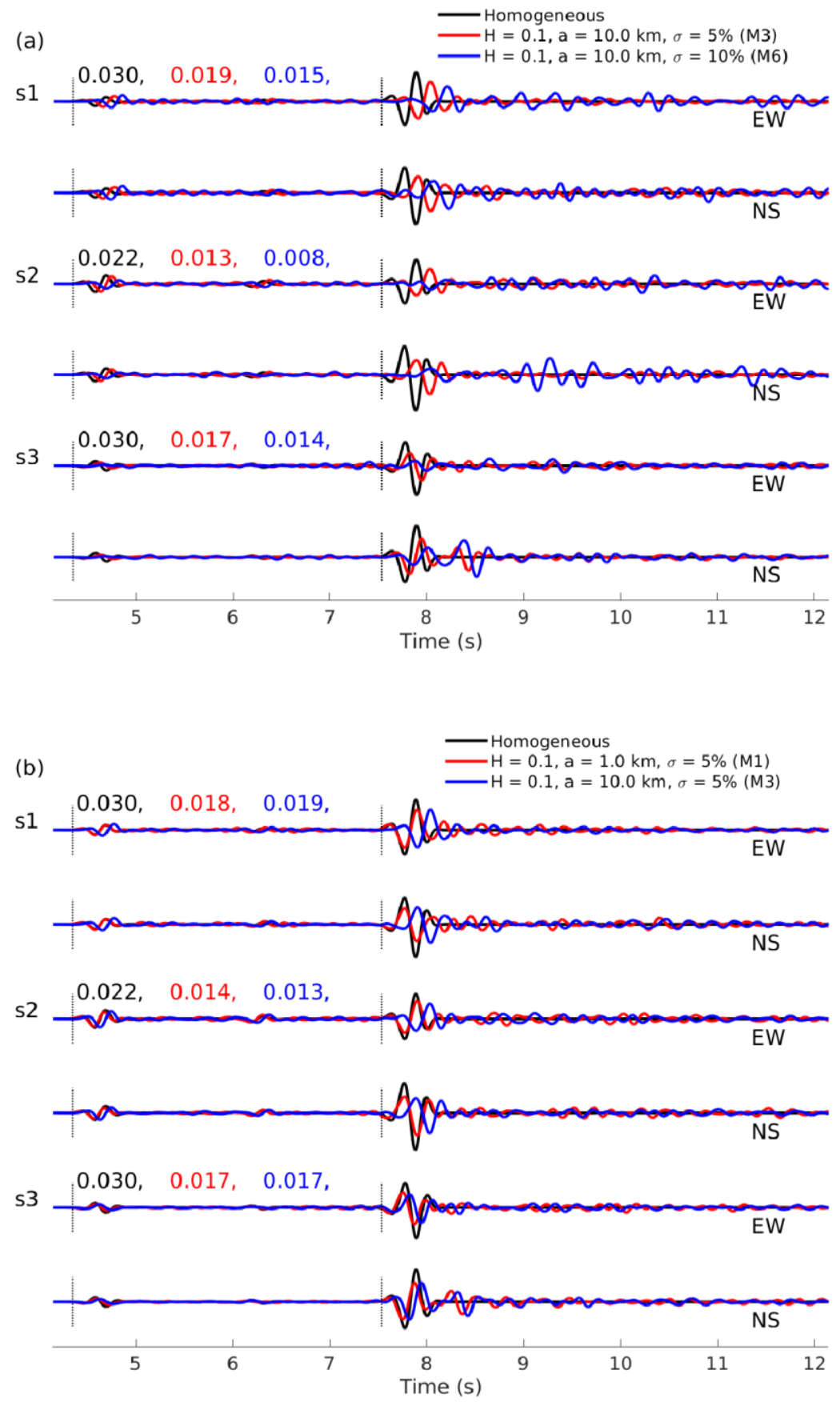

Figure 2: Horizontal components (East-West, EW, and North-South, NS) of ground acceleration $\left(\mathrm{m} / \mathrm{s}^{2}\right.$ ) at sites s1, s2, s3 (Fig 1a). Black dotted lines indicate theoretical P- and S-wave arrival times in the considered homogeneous medium. Color-coded numbers indicate PGA values at individual sites. Waveforms are normalized by their PGA-value in the homogeneous-medium simulations for a given site. (a) Illustration of scattering controlled by $\sigma$ for $k_{w} \cdot a \gg 1$ and small $H$; (b) Illustration of negligible effects of correlation length on scattering for $k_{w} \cdot a \gg 1$ and small $H$. 
(a)

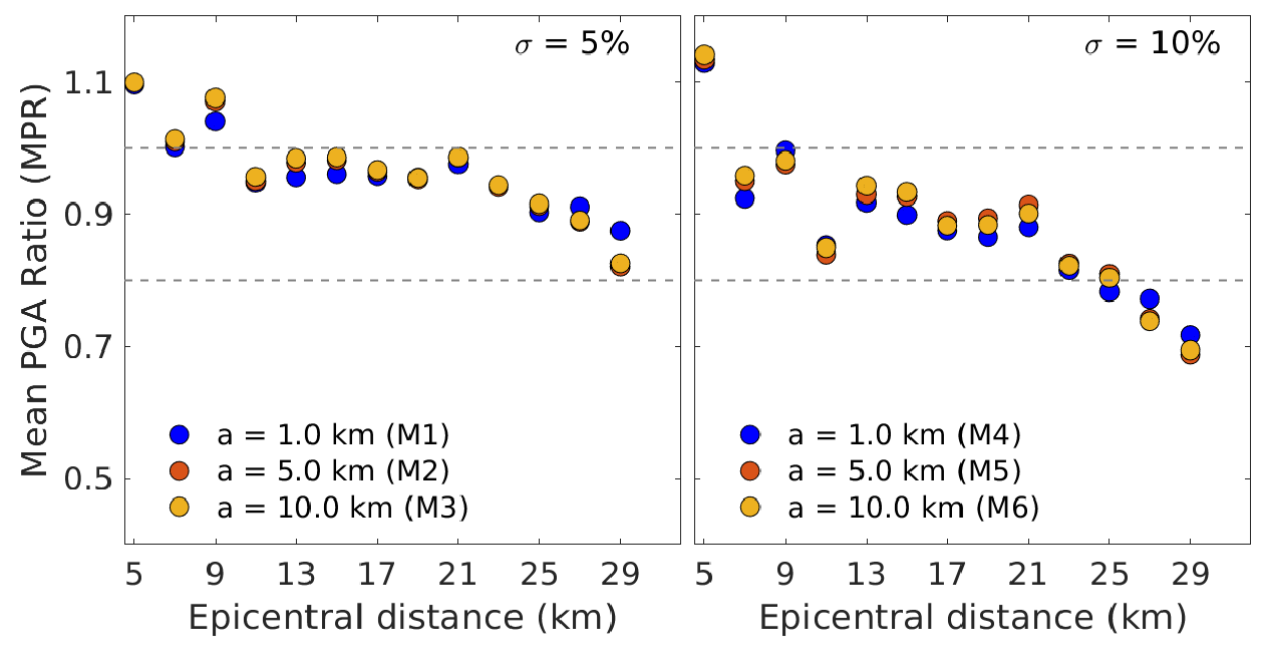

(b)

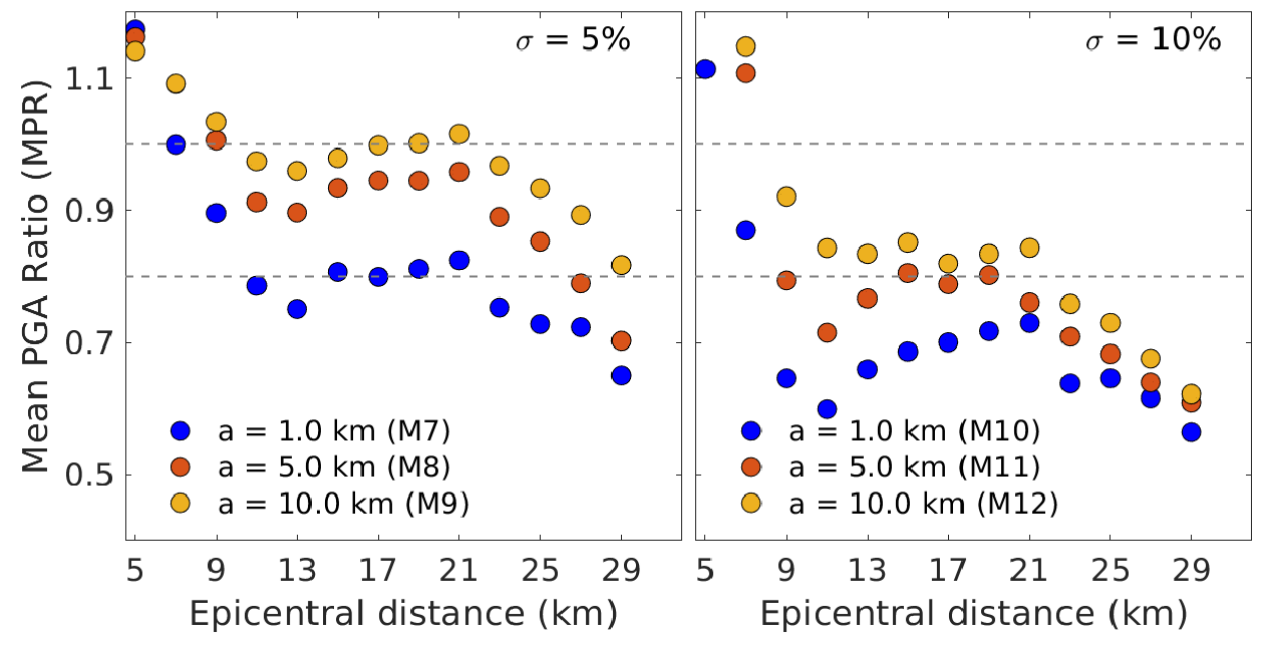

Figure 3: Mean PGA ratios (MPR) for all twelve numerical simulations as a function of distance, depicting the effects of wavefield scattering on ground-motions in the regime $k_{w} \cdot a \gg 1$. Panels (a) and (b) depict MPR for media with $\mathrm{H}=0.1$ and $\mathrm{H}=0.5$, respectively. Grey dashed lines are plotted to facilitate the MPR comparison in two nearby panels. Wavefield scattering is proportional to the standard deviation of medium heterogeneities, and inversely proportional to correlation length for large Hurst exponent $(H=0.5)$, but remains nearly unaffected by variations in correlation length for small Hurst exponent $(\mathrm{H}=0.1)$. The $k_{w} \cdot a$ maxima for correlation lengths of 1,5 and $10 \mathrm{~km}$ are $9.07,45.36$ and 90.72 , respectively. 
(a)

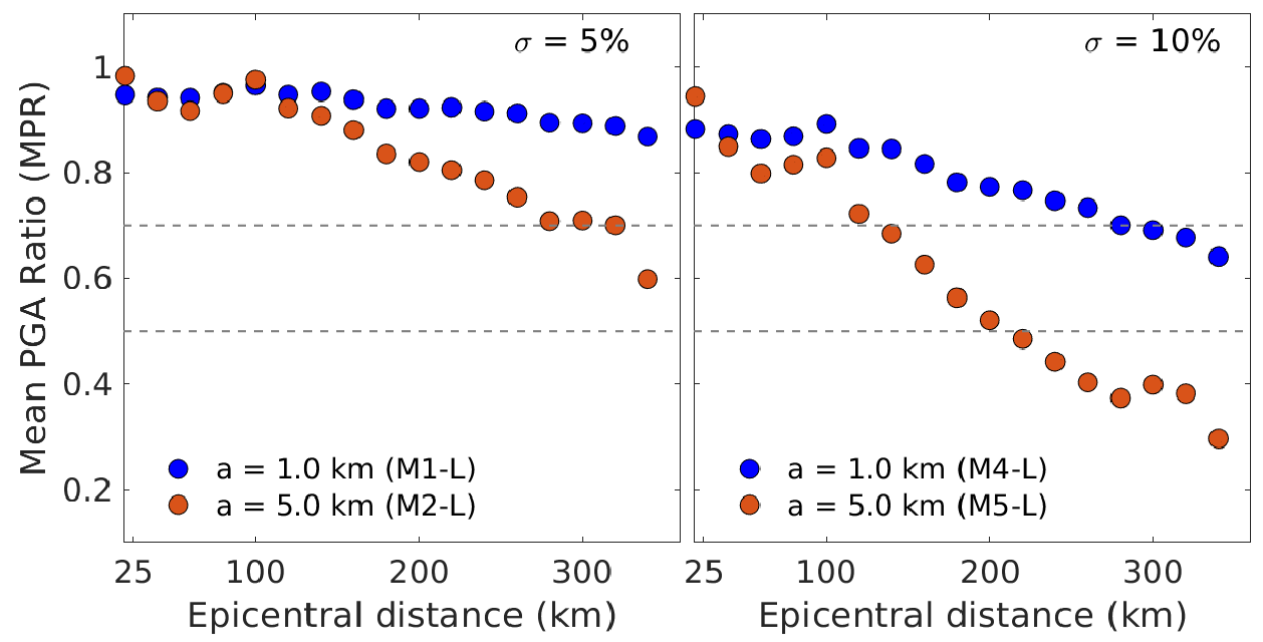

(b)

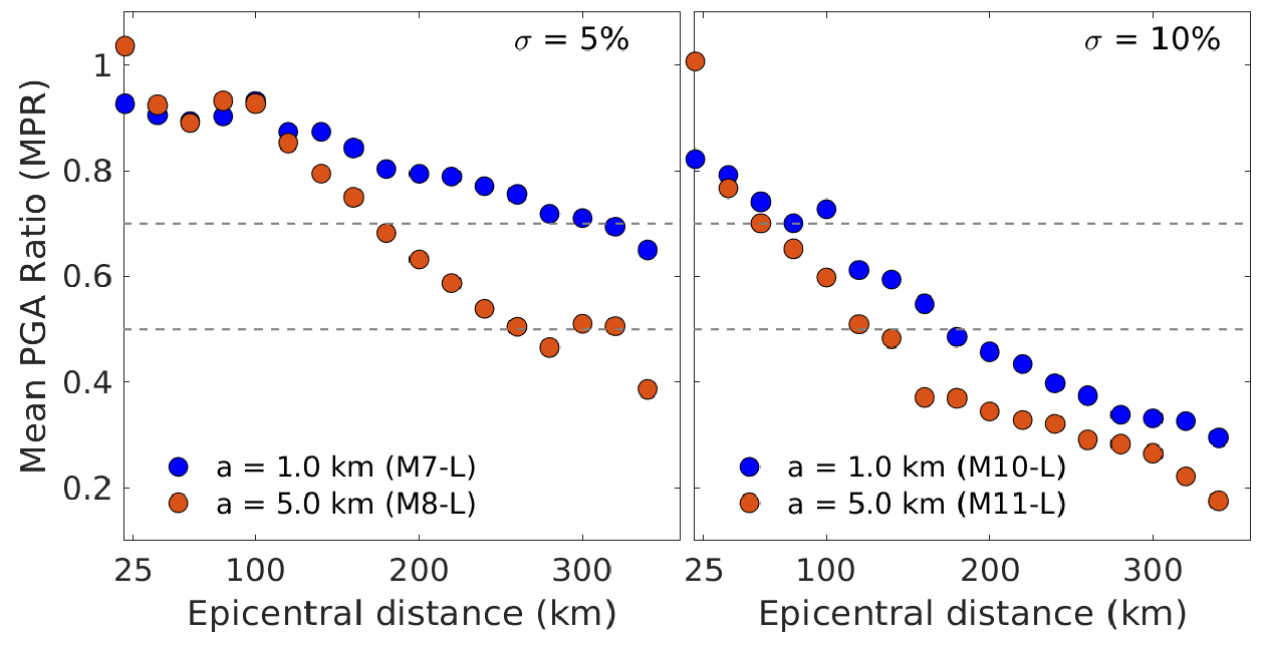

Figure 4: Mean PGA ratios (MPR) for eight numerical simulations as a function of distance, depicting the effects of wavefield scattering on ground-motions in the regime $k_{w} \cdot a \approx 1$. Panels (a) and (b) depict MPR for media with $\mathrm{H}=0.1$ and $\mathrm{H}=0.5$, respectively. Grey dashed lines are plotted to facilitate the MPR comparison in two nearby panels. Wavefield scattering is proportional to correlation length, Hurst exponent, and standard deviation of medium heterogeneities. The highest values of $k_{w} \cdot a$ for correlation lengths of 1 and $5 \mathrm{~km}$ are 0.90 and 4.53 , respectively. 
(a)

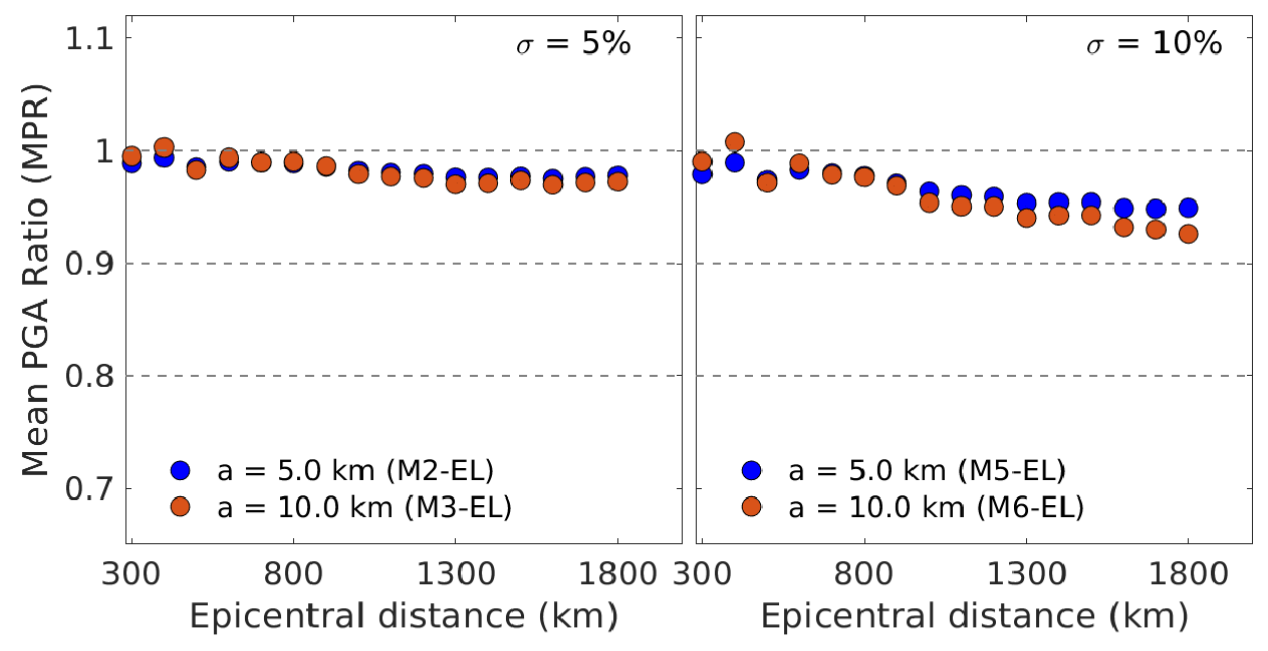

(b)

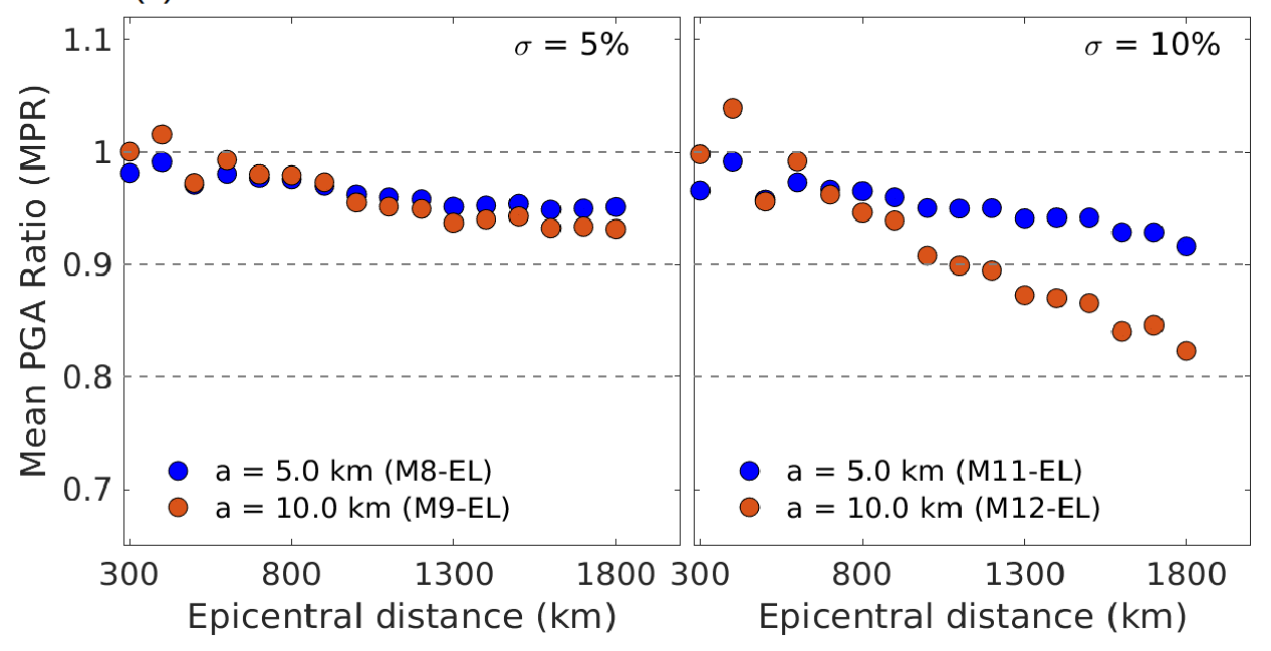

Figure 5: Mean PGA ratios (MPR) for all eight numerical simulations as a function of distance, depicting the effects of wavefield scattering on ground-motions in the regime $k_{w} \cdot a<1$. Panels (a) and (b) depict MPR for media with $\mathrm{H}=0.1$ and $\mathrm{H}=0.5$, respectively. Grey dashed lines are plotted to facilitate the MPR comparison in two nearby panels. Wavefield scattering is proportional to correlation length, Hurst exponent, and the standard deviation of medium heterogeneities. The highest values of $k_{w} \cdot a$ for correlation lengths of 5 and $10 \mathrm{~km}$ are 0.27 and 0.54 , respectively. 